\title{
AB INITIO FREE ENERGY CALCULATIONS OF THE SOLUBILITY OF SILICA IN METALLIC HYDROGEN AND APPLICATION TO GIANT PLANET CORES
}

\author{
F. González-Cataldo ${ }^{1}$, Hugh F. Wilson ${ }^{2,3}$, And B. Militzer ${ }^{2,4}$ \\ ${ }^{1}$ Grupo de NanoMateriales, Departamento de Física, Facultad de Ciencias, Universidad de Chile, Casilla 653, Santiago, Chile; fgonzalez@1pmd.cl \\ ${ }^{2}$ Department of Earth and Planetary Science, University of California Berkeley, Berkeley, CA 94720, USA \\ ${ }^{3}$ Virtual Nanoscience Laboratory, CSIRO Materials Science and Engineering, Parkville, Victoria 3052, Australia \\ ${ }^{4}$ Department Astronomy, University of California Berkeley, Berkeley, CA 94720, USA \\ Received 2014 January 6; accepted 2014 April 2; published 2014 May 6
}

\begin{abstract}
By combining density functional molecular dynamics simulations with a thermodynamic integration technique, we determine the free energy of metallic hydrogen and silica, $\mathrm{SiO}_{2}$, at megabar pressures and thousands of degrees Kelvin. Our ab initio solubility calculations show that silica dissolves into fluid hydrogen above $5000 \mathrm{~K}$ for pressures from 10 and 40 Mbars, which has implications for the evolution of rocky cores in giant gas planets like Jupiter, Saturn, and a substantial fraction of known extrasolar planets. Our findings underline the necessity of considering the erosion and redistribution of core materials in giant planet evolution models, but they also demonstrate that hot metallic hydrogen is a good solvent at megabar pressures, which has implications for high-pressure experiments.
\end{abstract}

Key words: planets and satellites: dynamical evolution and stability - planets and satellites: individual (Jupiter, Saturn) - planets and satellites: interiors

Online-only material: color figures

\section{INTRODUCTION}

Hydrogen is the simplest and most abundant atom in the universe, but its properties at high pressures remain poorly characterized (McMahon et al. 2012). In the interior of giant planets, hydrogen is predicted to occur in metallic form. While such a state has been generated at high temperatures with dynamic shock wave experiments (Weir et al. 1996), obtaining metallic hydrogen in static high-pressure experiments at room temperature has been an elusive goal. While the earliest theoretical estimates by Wigner \& Huntington (1935) placed an insulatorto-metal transition at only $25 \mathrm{GPa}$, recent advances in diamond anvil cell experiments (Eremets \& Troyan 2011; Howie et al. 2012; Zha et al. 2012) did not reveal conclusive evidence of metallization for pressures up to approximately $400 \mathrm{GPa}$. Consequently, the properties of metallic hydrogen have primarily been studied theoretically (Johnson \& Ashcroft 2000) and with ab initio computer simulations (Militzer \& Graham 2006; Vorberger et al. 2007; Hamel et al. 2011; McMahon et al. 2012; Morales et al. 2013a, 2013b; Soubiran et al. 2013; Goncharov et al. 2013; Becker et al. 2013) that we also use for this article, in which we focus on the interaction of metallic hydrogen and silica, $\mathrm{SiO}_{2}$. Silica is not only important for geophysics but also as a prototype material for studying condensed matter physics at extreme conditions. Hicks et al. (2006) used shock wave experiments to study the thermodynamic properties of liquid silica at megabar pressures.

In this article we focus on the question of whether silica dissolves into metallic hydrogen at megabar pressures, because this would have implications for the stability of the cores of solar and extrasolar gas giant planets. Many of the confirmed 800 extrasolar planets are gas giants that are primarily composed of dense fluid hydrogen and helium. Furthermore, the Kepler mission has detected 2740 planet candidates and has measured their radii and orbital periods within 22 months of observations (Batalha et al. 2013). In a few cases with multiple planets in close orbits, masses have been inferred from transit-timing variations
(Charbonneau et al. 2009). The Juno mission is scheduled to arrive at Jupiter in 2016 and will measure the gravitational field of our largest local gas giant with unprecedented accuracy, revealing clues about its inner mass distribution. Existing core-accretion models for gas giant formation (Mizuno et al. 1978) hold that these planets form from the rapid accretion of gas around a rock-ice protocore. Therefore, according to our understanding, the evolution of giant planets starts with a differentiated rocky core surrounded by an envelope of hot, dense hydrogen-helium gas. The temperature in the envelope rises, and the gravitational energy from accretion is converted to heat. An adiabatic temperature is rapidly established. The evolution of a giant planet is controlled by the energy loss due to thermal radiation (Fortney \& Nettelmann 2009). Conventional giant planet models assume a stable core and a sharp core-mantle boundary instead of taking into account the possibility that the metallic hydrogen layer may act as solvent for the initial protocore. Answering the question of whether giant planet cores remain stable on a billion year time scale may also provide an alternative explanation for the observed heavy element enrichment in giant planet atmospheres, which is currently attributed to late-arriving planetesimals (Niemann et al. 1996; Mahaffy et al. 1998). If a core dissolved, it would lead to double diffusive convection (Guillot et al. 2004; Stevenson 1982; Leconte \& Chabrier 2012, 2013) because gravity opposes the redistribution of heavy core materials. This would introduce compositional stratification and significantly reduce the rate at which heat can be transported out of the interior, with substantial implications for the thermal evolution and radius contraction of giant planets (Chabrier \& Baraffe 2007).

The initial cores of giant planets can be assumed to consist of a combination of rocky and icy materials. The rocky components are likely to be dominated by iron and magnesium silicate minerals. It was shown by Umemoto et al. (2006) that post-perovskite $\mathrm{MgSiO}_{3}$ separates into $\mathrm{MgO}$ and $\mathrm{SiO}_{2}$ beyond $\sim 10$ Mbars and $\sim 10,000 \mathrm{~K}$, which are conditions that are expected to be exceeded at the core-mantle boundaries of typical gas giant 
planets. Recent ab initio calculations predicted a substantial solubility of $\mathrm{MgO}$, water ice, and iron in fluid metallic hydrogen for the core-mantle boundary of Jupiter and Saturn (Wilson \& Militzer 2012b, 2012a; Wahl et al. 2013). Therefore, this study is focused on the solubility of remaining core material, $\mathrm{SiO}_{2}$, in order to obtain a more complete picture of the behavior of metallic hydrogen as a solvent of planetary materials.

At the core boundary of giant planets, the temperature and pressure conditions are estimated to be on the order of 10-40 Mbar and 10,000 to 20,000 K. Because such extreme conditions cannot yet be probed with laboratory experiments, we use ab initio computer simulations that can be used directly to characterized material at such $P-T$ conditions (Militzer \& Wilson 2010; Wilson \& Militzer 2010; Militzer 2013; Zhang et al. 2013).

\section{COMPUTATIONAL METHODS}

Using density functional molecular dynamics (DFT-MD), we calculated the Gibbs free energy of solvation, $\Delta G_{\text {sol }}$, of $\mathrm{SiO}_{2}$, given by the difference between the Gibbs free energy of the dissolved system and that of the separate compounds $\left(\mathrm{SiO}_{2}\right.$ and hydrogen) at fixed pressure-temperature conditions. We begin by computing the free energy of solvation of $\mathrm{SiO}_{2}$ for a mixing ratio of one solute atom per 128 hydrogen atoms (i.e., one $\mathrm{SiO}_{2}$ formula unit to $384 \mathrm{H}$ ) and later generalize our results to other concentrations.

$$
\begin{aligned}
\Delta G_{\mathrm{sol}}\left(\mathrm{SiO}_{2}: 384 \mathrm{H}\right) & =G\left(\mathrm{H}_{384} \mathrm{SiO}_{2}\right) \\
& -\left[G\left(\mathrm{H}_{384}\right)+G\left(\mathrm{SiO}_{2}\right)\right] .
\end{aligned}
$$

Given the large quantity of hydrogen gas in giant planets, we are primarily concerned with the low-concentration limit. Thus, we can assume that solute atoms do not interact with each other, and we introduce the following approximation:

$$
G\left(\mathrm{H}_{384} \mathrm{SiO}_{2}\right) \approx G\left(\mathrm{H}_{128} \mathrm{Si}\right)+2 G\left(\mathrm{H}_{128} \mathrm{O}\right)-k_{B} T \log (27 / 2) .
$$

The last term arises from the free energy of mixing because we do not have the same number of hydrogen atoms in each term.

Since the entropy term in the Gibbs free energies, $G=$ $E+P V-T S$, is not directly accessible in standard molecular dynamics simulations, we used a thermodynamic integration (TDI) technique (Morales et al. 2009; Wilson \& Militzer 2010, 2012a, 2012b; Militzer 2013; Sugino \& Car 1995) to compute the free energy difference between the system of interest and a simpler noninteracting system whose free energy may be computed explicitly. The difference in Helmholtz free energy between systems governed by two different potentials is given by

$$
\Delta F=\int_{0}^{1}\left\langle U_{2}-U_{1}\right\rangle_{\lambda} d \lambda,
$$

where the angle brackets denote an average taken over trajectories generated in the system governed by the hybrid potential energy function $U_{\lambda}$. This method provides a general scheme to calculate the Helmholtz free energy difference between two systems governed by potentials $U_{1}\left(\mathbf{r}_{i}\right)$ and $U_{2}\left(\mathbf{r}_{i}\right)$, connected by the hybrid potential $U_{\lambda}=(1-\lambda) U_{1}+\lambda U_{2}$. Since we have split the Gibbs free energy of solvation in energies of four different systems (pure silica, pure fluid hydrogen, fluid hydrogen with one $\mathrm{O}$ atom, and fluid hydrogen with one $\mathrm{Si}$ atom), we need to perform four separate sets of simulations at each pressure and temperature. Five equally spaced $\lambda$ values between 0 and 1
Table 1

Gibbs Free Energies of Pure Hydrogen, Hydrogen with Oxygen, Hydrogen with Silicon, and $\mathrm{SiO}_{2}$ (Solid in All Cases Except for $20 \mathrm{Mbar}$ and 20,000 K)

\begin{tabular}{lcrrr}
\hline \hline$P, T$ & $G\left(\mathrm{H}_{128}\right)$ & $\begin{array}{c}G\left(\mathrm{H}_{128} \mathrm{O}\right) \\
(\mathrm{eV})\end{array}$ & $\begin{array}{c}G\left(\mathrm{H}_{128} \mathrm{Si}\right) \\
(\mathrm{eV})\end{array}$ & \multicolumn{1}{c}{$\begin{array}{r}G\left(\mathrm{SiO}_{2}\right) \\
(\mathrm{eV})\end{array}$} \\
\hline 10,3 & $(\mathrm{eV})$ & $688.6(2)$ & $705.4(3)$ & $63.3(1)$ \\
10,5 & $671.4(2)$ & $573.5(4)$ & $590.5(5)$ & $60.2(1)$ \\
20,3 & $559.0(5)$ & $1304.7(2)$ & $1330.1(3)$ & $121.0(1)$ \\
20,5 & $1270.7(2)$ & $1202.4(5)$ & $1228.2(3)$ & $118.4(1)$ \\
20,10 & $1170.8(4)$ & $868.3(4)$ & $893.8(2)$ & $109.1(4)$ \\
20,20 & $843.2(4)$ & $26.9(6)$ & $53.6(7)$ & $81.8(1)$ \\
40,3 & $17.3(5)$ & $2191.8(2)$ & $2229.4(1)$ & $211.3(1)$ \\
40,7 & $2131.4(2)$ & $1991.4(3)$ & $2030.7(3)$ & $206.0(2)$ \\
40,10 & $1936.2(3)$ & $1799.0(6)$ & $1837.6(9)$ & $200.6(3)$ \\
40,15 & $1747.4(1)$ & $1427.9(4)$ & $1467.6(8)$ & $190.1(4)$ \\
\hline
\end{tabular}

are taken for each of them to get a smooth curve of $\left(U_{2}-U_{1}\right)$ versus $\lambda$ that can be interpolated via quadratic interpolation to determine the integral. Once we have obtained $\Delta F=F_{2}-F_{1}$, we add the known free energy $F_{1}$ to determine the energy of the system, $F_{2}$, governed by the potential $U_{2}$. The Gibbs free energy is obtained by the addition of the $P V$ term.

The TDI is performed in two steps: first from a system governed by DFT forces to a system interacting via a classical pair potential and then from the classical system to a reference system with a free energy that is known analytically. For fluids, we chose an ideal gas, while for solid systems we selected a system of independent harmonic oscillators as the reference system. For the fluid systems, we constructed the classical twobody potentials by fitting to the forces of a DFT-MD trajectory using the force-matching methodology (Izvekov et al. 2004; Tangney \& Scandolo 2002). In the case of a solid $\mathrm{SiO}_{2}$ system, we first determined the harmonic spring constants from mean squared displacement from an atom's lattice site and then fit the residual forces with pair potentials.

The DFT calculations throughout this work were performed using the VASP code (Kresse \& Furthmüller 1996). We used pseudopotentials of the projector-augmented wave type (Blochl 1994), the exchange-correlation functional of Perdew, Burke, and Ernzerhof (Perdew et al. 1996), a cutoff energy of $900 \mathrm{eV}$ for the plane wave expansion of the wavefunctions, and a $2 \times 2 \times 2$ $k$-point grid to sample the Brillouin zone, except for the $\mathrm{SiO}_{2}$ simulations where we used only the $\Gamma$ point. An MD time step of 0.2 fs was used, and the simulation time ranged between 0.5 and $2.0 \mathrm{ps}$. The $\Delta G_{\text {sol }}$ values were confirmed to be well converged with respect to these parameters for the purpose of this dissolution calculation.

For pure solid $\mathrm{SiO}_{2}$, we used the $\mathrm{Fe}_{2} \mathrm{P}$-type structure with the space group $\mathrm{P}-62 \mathrm{~m}$ that was recently predicted by Tsuchiya $\&$ Tsuchiya (2011) to be the ground-state structure at pressures above $7 \mathrm{Mbar}$. We analyzed the stability of each material phase at pressures ranging from 10 to $40 \mathrm{Mbar}$ and temperatures ranging from 3000 to $20,000 \mathrm{~K}$ and confirmed that the structure remained solid for all cases under consideration except for $20,000 \mathrm{~K}$ and $20 \mathrm{Mbar}$, where we found liquid $\mathrm{SiO}_{2}$ to be the stable phase.

Gibbs energies were computed for the following system sizes: $\mathrm{H}_{128}, \mathrm{H}_{128} \mathrm{Si}, \mathrm{H}_{128} \mathrm{O}$, solid $\mathrm{SiO}_{2}$ in a 72 atom supercell, and liquid $\mathrm{SiO}_{2}$ in a cubic cell with 96 atoms. The resulting Gibbs free energies are shown in Table 1 and plotted as a function of temperature in Figure 1. The error bars on the $G$ values are dominated by two terms, the more significant one being the uncertainty in the volume at the desired pressure due to 




Figure 1. Computed solvation curve $\Delta G$ for different pressures, where a negative Gibbs free energy represents a preference for the dissolved state. Open squares, circles, and diamonds represent states where $\mathrm{SiO}_{2}$ is solid, while the filled red circle corresponds to a state where $\mathrm{SiO}_{2}$ was found to be liquid.

(A color version of this figure is available in the online journal.)

Table 2

Gibbs Free Energies of Solubility for $\mathrm{SiO}_{2}$ into Hydrogen at a Concentration of One Part in 384 Hydrogen Atoms

\begin{tabular}{|c|c|c|}
\hline $\begin{array}{l}P \\
\text { (Mbar) }\end{array}$ & $\begin{array}{c}T \\
(\mathrm{~K})\end{array}$ & $\begin{array}{c}\Delta G_{\text {sol }} \\
(\mathrm{eV})\end{array}$ \\
\hline 10 & 3000 & $4.92 \pm 0.69$ \\
\hline 10 & 5000 & $0.32 \pm 1.90$ \\
\hline 20 & 3000 & $6.35 \pm 0.78$ \\
\hline 20 & 5000 & $2.18 \pm 1.63$ \\
\hline 20 & 10000 & $-8.37 \pm 1.45$ \\
\hline 20 & 20000 & $-26.47 \pm 2.14$ \\
\hline 40 & 3000 & $7.40 \pm 0.63$ \\
\hline 40 & 7000 & $-0.93 \pm 1.19$ \\
\hline 40 & 10000 & $-7.13 \pm 1.60$ \\
\hline 40 & 15000 & $-19.59 \pm 2.55$ \\
\hline
\end{tabular}

finite simulation time and the other being the uncertainty in the $\left\langle U_{\mathrm{DFT}}-U_{\text {classical }}\right\rangle$ terms in the TDI.

\section{RESULTS AND DISCUSSION}

The results in Table 1 are used in conjunction with the Equations (1) and (2) to obtain the Gibbs free energies of solvation in Table 2. A negative Gibbs free energy implies that the dissolved state has a lower Gibbs free energy than the separate phases, demonstrating that solvation is preferred at a concentration of 1:384. A positive free energy indicates that the fluid system is supersaturated and that deposition of fluid $\mathrm{SiO}_{2}$, or formation of solid grains, will be thermodynamically favored. Our results in Figure 2 are not too different from those found for $\mathrm{MgO}$ (Wilson \& Militzer 2012a), showing that $\mathrm{SiO}_{2}$ is also soluble at temperatures that are a bit lower for a given pressure, certainly well below those at Jupiter's core-mantle boundary. This solubility occurs at higher temperatures than water ice (Wilson \& Militzer 2012b), where the solubility was already strongly favored at much lower temperatures of 2000-3000 K in the same pressure range.

Gibbs free energy of solvation can be generalized to other concentrations without performing additional DFT-MD simulations if the average separation between solute atoms is large enough so that their interaction can be neglected. Under this assumption, the free energy of mixing yields the difference of



Figure 2. Saturation solubility of $\mathrm{SiO}_{2}$ in metallic hydrogen (this work; solid lines) and $\mathrm{MgO}$ (from Wilson \& Militzer 2012a; dashed lines) as a function of temperature and pressure. The temperature-pressure conditions of the Jovian and Saturnian core-mantle boundaries are shown for comparison.

(A color version of this figure is available in the online journal.)

$\Delta G_{\text {sol }}$ between a solution of one $\mathrm{SiO}_{2}$ formula unit in $m$ versus $n$ hydrogen atoms (Wilson \& Militzer 2012b),

$$
\begin{aligned}
\frac{\Delta G_{\mathrm{sol}}[m]-\Delta G_{\mathrm{sol}}[n]}{k_{B} T}= & m \ln \left(\frac{m V_{\mathrm{H}}+V_{\mathrm{S} i}+2 V_{\mathrm{O}}}{m V_{\mathrm{H}}}\right) \\
& -n \ln \left(\frac{n V_{\mathrm{H}}+V_{\mathrm{S} i}+2 V_{\mathrm{O}}}{n V_{\mathrm{H}}}\right) \\
& +3 \ln \left(\frac{m V_{\mathrm{H}}+V_{\mathrm{S} i}+2 V_{\mathrm{O}}}{n V_{\mathrm{H}}+V_{\mathrm{S} i}+2 V_{\mathrm{O}}}\right),
\end{aligned}
$$

where $V_{\mathrm{H}}, V_{\mathrm{O}}$, and $V_{\mathrm{Si}}$ are the effective volumes of the $\mathrm{H}$, $\mathrm{O}$, and $\mathrm{Si}$ atoms that we obtained by comparing the volumes of the different fluid simulations at the same pressure and temperature. Using a linear interpolation for the data in Table 2, we can determine the saturation concentration for $\mathrm{SiO}_{2}$ in fluid hydrogen as a function of temperature and pressure throughout the 10-40 Mbar and 3000-20,000 K range. A contour plot of constant saturation solubility is shown in Figure 2. Solute concentrations higher than 1:100 are not shown because they may lead to interactions between solute atoms. Despite error bars of approximately $1000 \mathrm{~K}$ that should be considered to be uncertainties of the contours, these results show that $\mathrm{SiO}_{2}$ is highly soluble at both Jupiter's and Saturn's core-mantle boundary conditions. This is in contrast to $\mathrm{MgO}$, which may be not be as highly soluble at Saturnian core conditions. Therefore, there exists the possibility that $\mathrm{SiO}_{2}$ may dissolve from Saturn's core but leave solid $\mathrm{MgO}$ behind.

The Gibbs free energy of solubility $\Delta G$ may be split into three components: an internal energy component $\Delta U$, a volume contribution $P \Delta V$, and an entropic term $-T \Delta S$, which lead to $\Delta G=\Delta U-T \Delta S+P \Delta V$. The $P \Delta V$ and $\Delta U$ values can be directly extracted from standard DFT-MD simulations. The remaining term is $-T \Delta S$ from calculated $\Delta F$. All terms are shown in Figure 3 as a function of temperature at 20 Mbar. 


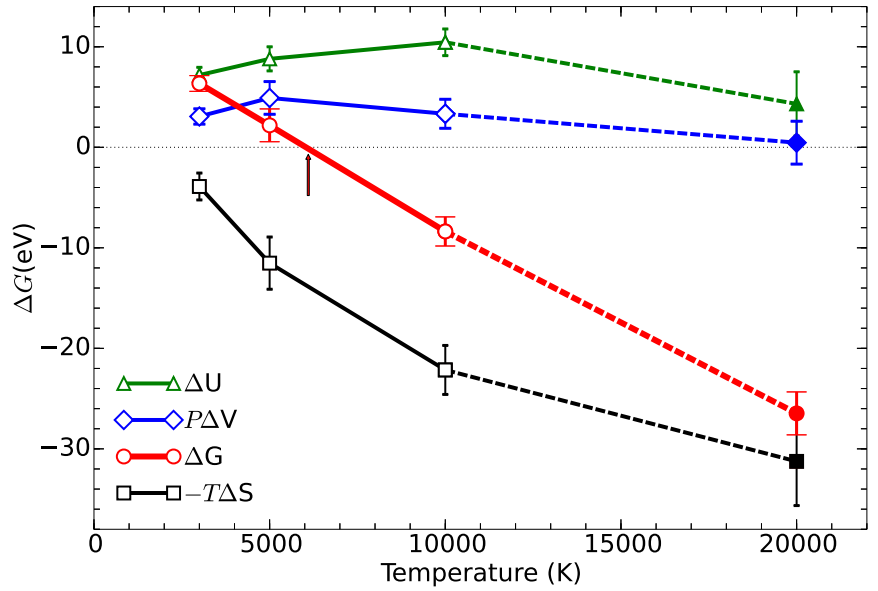

Figure 3. Splitting of $\Delta G$ at 20 Mbar into its three constituent components: the internal energy term $\Delta U$ that represents differences in chemical binding, the $P \Delta V$ term that arises from volume differences, and the remaining $-T \Delta S$ term that represents entropic effects. The arrow denotes the temperature above which dissolutions are favored at the concentration of one $\mathrm{SiO}_{2}$ in 384 hydrogen atoms.

(A color version of this figure is available in the online journal.)

The $P \Delta V$ term was comparatively close to zero but shows a slight preference of approximately $0.5-3.5 \mathrm{eV}$ for materials to remain separate, suggesting the dissolution reaction is not a pressure-driven process. The $\Delta U$ term also shows an energy barrier against dissolution that is slightly larger than the $P \Delta V$ term. For all temperatures under consideration, the entropic term $-T \Delta S$ is negative, confirming that more disorder is present in the dissolved state. The $-T \Delta S$ term exhibits a steep negative slope as a function of temperature that introduces a sign change into the Gibbs energy balance at $T=6000 \mathrm{~K}$ for $20 \mathrm{Mbar}$ where $\mathrm{SiO}_{2}$ is solid. Above this temperature, dissolution is favored, which can be considered to be an entropy-driven process similar to $\mathrm{H}_{2} \mathrm{O}$ and $\mathrm{MgO}$ (Wilson \& Militzer 2012b, 2012a). For iron, the $-T \Delta S$ term also favors dissolution, but there is no energy barrier to overcome because at megabar pressures, hydrogen and iron are both metals that mix at low temperatures (Wahl et al. 2013).

Figure 3 shows that the Gibbs free energy of solubility depends linearly on temperature. This trend continues into the liquid phase, as our $20,000 \mathrm{~K}$ data point confirms. For the temperature interval from 10,000 to $20,000 \mathrm{~K}$, where one expects $\mathrm{SiO}_{2}$ to melt at $20 \mathrm{Mbar}$ (González-Cataldo et al. 2014), this trend implies that the Gibbs free energy difference between the solid and liquid phases is small compared with the Gibbs free energy change induced by dissolution. If $\mathrm{SiO}_{2}$ melts in the vicinity of the dissolution transition, one would expect this transition to introduce only a modest change in slope into saturation solubility curves in Figure 2 because the Gibbs free energy changes continuously across the melting transition.

\section{CONCLUSIONS}

The presented ab initio free energy calculations demonstrate that metallic hydrogen is a good solvent for silica at megabar pressures for temperatures above $5000 \mathrm{~K}$. This result is consistent with recent $\mathrm{ab}$ initio solubility calculations that predicted $\mathrm{H}_{2} \mathrm{O}$ to dissolve into metallic hydrogen at 2000-3000 K (Wilson \& Militzer 2012b) and $\mathrm{MgO}$ at 6000-8000 K (Wilson \& Militzer 2012a). This suggests other insulating materials may dissolve at a comparable temperature range. Iron was found to dissolve at low temperatures because it is a metal (Wahl et al. 2013). These findings suggest that hydrogen will spontaneously react with any material that is used as confinement during dynamic shock wave experiments that reach megabar pressures and high temperatures. Our findings indirectly place a limit on the time scale of such experiments before a significant contamination of the sample sets in.

Our results also have implications for the evolution of giant planets. We predict that the $\mathrm{SiO}_{2}$ component has been eroded from the cores of Jupiter and Saturn, while MgO in Saturn's core may remain stable. Therefore, a partial solvation of the Saturnian core could have taken place, taking away more volatile materials like $\mathrm{SiO}_{2}$ and water ice and leaving behind less soluble materials like $\mathrm{MgO}$. Because of the differences between the solubility curves of $\mathrm{MgO}$ and $\mathrm{SiO}_{2}$ in Figure 2, partial core erosion may also occur in extrasolar gas giant planets that are smaller than Saturn but still large enough to contain metallic hydrogen. In general, larger and hotter interiors are expected to promote core erosion and a greater degree of redistribution of heavy material (Guillot et al. 2004). Provided the necessary energy for convection, the material may be redistributed throughout the entire planet, leading to an enrichment in heavy elements in giant planet atmospheres that have previously been attributed to late-arriving planetesimals.

Alternatively, the rate of redistribution may be hampered by compositional stratification that is the result of double diffusion convection (Guillot et al. 2004; Stevenson 1982; Leconte \& Chabrier 2012). The stratification would also limit the heat transport from the core, delay a planet's cooling, and possibly explain the inflated radii that have been observed for a large of number giant exoplanets (Chabrier \& Baraffe 2007).

We have assumed that there is sufficient hydrogen available for the approximation of noninteracting solute atoms to remain valid. Also, other stoichiometries of $\mathrm{SiO}_{2}$ have not been considered, assuming $\mathrm{Si}$ and $\mathrm{O}$ to dissolve in a one-to-two ratio according to the charge balance.

Our results confirm that the core erosion must be taken into account when future models of giant planet interiors are constructed. The redistribution of heavy elements has important implications in the heat transport and mass distribution, and core erosion plays a fundamental role in this aspect since it may be the source of the presence of these elements in the outer layers. Further models for the upconvection of core material are also necessary to understand the present structure of Jupiter and other planets, whose effects may be reflected on the gravitational moments to be measured by the Juno mission.

F.G.-C. acknowledges CONICYT PhD fellowship No. 201090712 and a short-term fellowship from Universidad de Chile, Chile. H.F.W. and B.M. received support from NASA and NSF.

\section{REFERENCES}

Batalha, N. M., Rowe, J. F., Bryson, S. T., et al. 2013, ApJS, 204, 24 Becker, A., Nettelmann, N., Holst, B., \& Redmer, R. 2013, PhRvB, 88, 045122 Blochl, P. E. 1994, PhRvB, 50, 17953

Chabrier, G., \& Baraffe, I. 2007, ApJL, 661, L81

Charbonneau, D., Berta, Z. K., Irwin, J., et al. 2009, Natur, 462, 891

Eremets, M. I., \& Troyan, I. A. 2011, NatMa, 10, 927

Fortney, J. J., \& Nettelmann, N. 2009, SSRv, 152, 423

Goncharov, A. F., Tse, J. S., Wang, H., et al. 2013, PhRvB, 87, 024101

González-Cataldo, F., Davis, S., \& Gutiérrez, G. 2014, PNAS, submitted

Guillot, T., Stevenson, D. J., Hubbard, W. B., \& Saumon, D. 2004, in The interior of Jupiter, ed. F. Bagenal, T. E. Dowling, \& W. B. McKinnon (Cambridge: Cambridge Univ. Press), 35 
Hamel, S., Morales, M. A., \& Schwegler, E. 2011, PhRvB, 84, 165110

Hicks, D. G., Boehly, T. R., Eggert, J. H., et al. 2006, PhRvL, 97, 025502

Howie, R. T., Guillaume, C. L., Scheler, T., Goncharov, A. F., \& Gregoryanz, E. 2012, PhRvL, 108, 125501

Izvekov, S., Parrinello, M., Burnham, C. J., \& Voth, G. A. 2004, JChPh, 120,10896

Johnson, K. A., \& Ashcroft, N. W. 2000, Natur, 403, 632

Kresse, G., \& Furthmüller, J. 1996, PhRvB, 54, 11169

Leconte, J., \& Chabrier, G. 2012, A\&A, 540, A20

Leconte, J., \& Chabrier, G. 2013, NatGe, 6, 347

Mahaffy, P. R., Niemann, H. B., Alpert, A., et al. 1998, BAAS, 30, 1066

McMahon, J. M., Morales, M. A., Pierleoni, C., \& Ceperley, D. M. 2012, RvMP, 84, 1607

Militzer, B. 2013, PhRvB, 87, 014202

Militzer, B., \& Graham, R. L. 2006, JPCS, 67, 2136

Militzer, B., \& Wilson, H. F. 2010, PhRvL, 105, 195701

Mizuno, H., Nakazawa, K., \& Hayashi, C. 1978, PThPh, 60, 699

Morales, M. A., Hamel, S., Caspersen, K., \& Schwegler, E. 2013a, PhRvB, 87, 174105

Morales, M. A., McMahon, J. M., Pierleoni, C., \& Ceperley, D. M. 2013b, PhRvB, 87, 184107
Morales, M. A., Schwegler, E., Ceperley, D., et al. 2009, PNAS, 106, 1324

Niemann, H. B., Atreya, S. K., Carignan, G. R., et al. 1996, Sci, 272, 846

Perdew, J. P., Burke, K., \& Ernzerhof, M. 1996, PhRvL, 77, 3865

Soubiran, F., Mazevet, S., Winisdoerffer, C., \& Chabrier, G. 2013, PhRvB, 87,165114

Stevenson, D. J. 1982, P\&SS, 30, 755

Sugino, O., \& Car, R. 1995, PhRvL, 74, 1823

Tangney, P., \& Scandolo, S. 2002, JChPh, 117, 8898

Tsuchiya, T., \& Tsuchiya, J. 2011, PNAS, 108, 1252

Umemoto, K., Wentzcovitch, R. M., \& Allen, P. B. 2006, Sci, 311, 983

Vorberger, J., Tamblyn, I., Militzer, B., \& Bonev, S. 2007, PhRvB, 75, 024206

Wahl, S. M., Wilson, H. F., \& Militzer, B. 2013, ApJ, 773, 95

Weir, S., Mitchell, A., \& Nellis, W. 1996, PhRvL, 76, 1860

Wigner, E., \& Huntington, H. B. 1935, JChPh, 3, 764

Wilson, H., \& Militzer, B. 2012a, PhRvL, 108, 111101

Wilson, H. F., \& Militzer, B. 2010, PhRvL, 104, 121101

Wilson, H. F., \& Militzer, B. 2012b, ApJ, 745, 54

Zha, C. S., Liu, Z., \& Hemley, R. J. 2012, PhRvL, 108, 146402

Zhang, S., Wilson, H. F., Driver, K. P., \& Militzer, B. 2013, PhRvB, 87, 024112 